\title{
Serious about C-ERAS (Cardiac ERAS)
}

\author{
Sanjeet Singh'*, Sudeep Das De', Ahmad Al-Adhami', Yasser Hegazy', Kirsty Graham², Giuseppe Bozzetti \\ Fraser Sutherland', Philip Curry' ', Nawwar Al-Attar', Zahid Mahmood' \\ 'Department of Cardiothoracic Surgery, Golden Jubilee National Hospital, UK \\ ${ }^{2}$ Enhanced Recovery Nurse Practitioner, Golden Jubilee National Hospital, UK \\ ${ }^{3}$ Department of Anaesthesia and Critical Care, Golden Jubilee National Hospital, UK
}

Article Info

\section{Article Notes}

Received: March 16, 2019

Accepted: April 16, 2020

\section{${ }^{*}$ Correspondence:}

Dr. Sanjeet Singh, MBChB(Edin) MSc(Edin) MRCS(Edin)

PhD (Glasgow), Cardiac Transplant Research Fellow,

Golden Jubilee National Hospital, United Kingdom; Email: sanjeetsingh@nhs.net.

(C) 2020 Singh S. This article is distributed under the terms of the Creative Commons Attribution 4.0 International License.

\section{Abstract}

Enhanced Recovery After Surgery (ERAS) incorporates multi-modal interventions that synergistically improve patient outcome. Its goals include improving patients functionally pre-operatively, reducing the stress of surgery intra-operatively to facilitate early return to daily activities. We conducted a pilot study at our unit recruiting patients undergoing elective coronary artery bypass grafting (CABG) into the Cardiac ERAS (C-ERAS) pilot and compared them with the patients undergoing CABG meeting the ERAS criteria but who not included the C-ERAS pilot (Control).

Materials and Methods: 122 C-ERAS patients were compared to 91 control patients who underwent CABG only from the period of July 2015 to September 2016. All C-ERAS patients received pre-operative counselling by a dedicated ERAS Practitioner to manage expectations of the patient journey, health promotion and pre-operative optimisation advice. Emphasis was made on educating the patient on daily goals for recovery and patients were followed up daily by the ERAS practitioner post-operatively.

Results: There were 122 patients in the C-ERAS group and 91 patients in the control group. The mean age was $63.6 \pm 9.9$ years. $181(85 \%)$ of the patients were males. After adjusting for the abovementioned confounders, C-ERAS patients had a shorter length of stay that was statistically significant. ( 2.36 days shorter ( $95 \% \mathrm{Cl} ; 1.01-3.7$ days; $p<0.01$ ). The difference in mean bed day costs was $£ 1153.70(95 \% \mathrm{Cl}, £ 553.70-£ 1753.7 ; p<0.01)$ less in the C-ERAS cohort.

Conclusion: This study highlighted that C-ERAS is a safe and feasible pathway to reduce in-hospital stay with no difference in complications and readmission rates compared to routine management of patients. There was also a significant cost saving with the C-ERAS pathway mimicking the results in enhanced recovery programmes in the other surgical specialities.

\section{List of Abbreviations}

C-ERAS: Cardiac Enhanced Recovery After Surgery

CABG: Coronary Artery Bypass Grafting

BMI: Body Mass Index

NYHA: New York Heart Association Functional Classification of Symptoms

CCS: Canadian Cardiovascular Society grading of angina pectoris

\section{LV: Left Ventricle}

ICU: Intensive Care Unit

\section{Introduction}

Enhanced Recovery after surgery was a concept introduced by 
Henrik Kehlet ${ }^{1}$ with protocols aimed to ameliorate the stress response to surgery by employing multimodal means with a summation of gains to optimise post-operative recovery. These processes begin in the pre-operative phase with a view to reduce the length of stay, promote earlier mobilisation, empower patients to be a part of their own recovery, and reduce morbidity and post-operative pain as demonstrated by a multitude of randomised trials ${ }^{2-6}$ and meta-analyses ${ }^{7-10}$ across the surgical specialties, thereby reshaping models of care.

Key outcomes measured in most ERAS programmes include patient satisfaction scores, reduction in hospital costs and an earlier return to pre-operative function when compared to conventional surgical pathways.

Despite the ubiquity of ERAS across the surgical specialties, there is a scarcity in data for ERAS post-cardiac surgery. Extrapolation of data from other specialties have enabled the formation of an ERAS programme (C-ERAS) at our unit.

C-ERAS is currently implemented to both elective and emergency patients following its evolvement in other surgical specialties ${ }^{11}$. Since its implementation, over 100 patients have benefited from this pathway. We undertook a pilot study to look at the early outcomes of the C-ERAS programme at the largest cardiac surgical unit in Scotland.

\section{Materials and Methods}

A prospectively collected local cardiothoracic database was retrospectively interrogated. 122 C-ERAS patients were compared to 91 control patients who underwent CABG only from the period of July 2015 to September 2016. All C-ERAS patients received pre-operative counselling by a dedicated ERAS Practitioner to manage expectations of the patient journey, health promotion and pre-operative optimisation advice. Emphasis was made on educating the patient on daily goals for recovery and patients were followed up daily by the ERAS practitioner post-operatively. They are also given a C-ERAS booklet and shown a video delineating the care pathway of the patient. The control group received education but did not have this focused support. The study was approved by the clinical governance department (ID: 1427).s

C-ERAS patients were seen by a dedicated physiotherapist on admission to reiterate the postoperative exercises.

\section{Intraoperatively}

Both groups underwent routine induction as per anaesthetist preference. Cardiopulmonary bypass was instituted by normothermia $\left(37^{\circ} \mathrm{C}\right)$ for the C-ERAS patients and mild hypothermia $\left(32^{\circ} \mathrm{C}\right)$ in the control group. Cardioplegia administration, termination of bypass and administration of protamine were similar in both groups.

\section{Post-operatively}

C-ERAS patients were extubated within 6 hours postsurgery by Nurse-led extubation under the supervision of the duty intensivist. Opioid infusions, IV paracetamol and IV fluids were discontinued when oral intake established (<12 hours postoperatively). Post-operative mobilisation was encouraged in the C-ERAS group. This included being up to sit in a chair 12 hours post-surgery and mobilising within 24 hours postoperatively.

Patients in the control group were extubated when appropriate with IV infusions stopped as per direction of the respective surgeons. IV fluids and infusions were stopped 24 hours post-operatively or as per surgeons' instructions.

C-ERAS patients who met the discharge criteria from ICU were discharged directly to the wards bypassing the High Dependency Unit (HDU). The protocol is as follows.

- Patient has been up to sit in chair

- Diet established

- Stable blood sugars

- Kardex reviewed and updated

- Satisfactory pain control established - oral analgesia (4 doses post-op Modified Release Oxycodone)

- Drains removed

- $\mathrm{Hb}>8$

- Creatinine within normal range for patient

- Urine output $>30 \mathrm{ml} / \mathrm{hr}$ (or $>40 \mathrm{ml} / \mathrm{hr}$ in patients $>80 \mathrm{~kg}$ )

- Stable rhythm - not pacing dependant

- No inotropic support

- Arterial line removed

- Central line in situ, capped off.

- Fluid balance to continue, catheter in-situ

- No cognitive impairment

- Weaning 02 and decreasing requirements

Outcomes measured were length of hospital stay, bed costs, post-operative complications (new onset Atrial Fibrillation requiring medical treatment, Acute Kidney Injury (with a raised creatinine level of more than 1.5 from the preoperative level ${ }^{12}$; respiratory failure (requiring non-invasive ventilation or reintubation, myocardial infarction, cerebrovascular event, hospital acquired infections and death.

\section{Statistical Analysis}

Comparison of patient characteristics between 
groups were made using the 2 sample students t-test for parametric continuous variables, Mann-Whitney $\mathrm{U}$ test for non-parametric continuous variables and the $\chi^{2}$-test for categorical variables. A multivariate linear regression model was used to compare the differences in the length of stay and bed-day costs, the potential confounders that were adjusted for in our study include: Euroscore, age at operation, smoking status, gender, BMI, NYHA class, Canadian Cardiovascular Society (CCS) angina classification, diabetes mellitus, urgent/ elective procedures and left ventricular function to eliminate potential confounding differences in the patient populations within the 2 cohorts.

\section{Results}

There were 122 patients in the C-ERAS group and 91

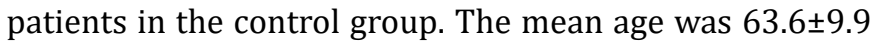
years. $181(85 \%)$ of the patients were males.

After adjusting for the abovementioned confounders, C-ERAS patients had a shorter length of stay that was statistically significant. (2.36 days shorter (95\% CI; 1.01 3.7 days; $p<0.01$ ). The difference in mean bed day costs was $£ 1153.70$ ( $95 \% \mathrm{CI}, £ 553.70-£ 1753.7 ; \mathrm{p}<0.01$ ) less in the C-ERAS cohort.

Table 1. Patient Demographics of the C-ERAS and Control Groups (unadjusted)

\begin{tabular}{|l|c|c|c|}
\hline \multicolumn{1}{|c|}{ Details } & C-ERAS $(\mathbf{n = 1 2 2})$ & Control $(\mathbf{n}=\mathbf{9 1})$ & p-Value \\
\hline Age (years) & $66.7 \pm 9.9$ & $61.2 \pm 9.4$ & 0.000 \\
\hline Sex & & & \\
Male & 111 & 91 & \\
Female & 11 & 15 & 0.302 \\
\hline BMI(kg/m $\left.{ }^{2}\right)$ & $29.17 \pm 4.4$ & $30.6 \pm 6.0$ & 0.241 \\
\hline Smoking & 7 & 15 & 0.017 \\
\hline NYHA class of heart failure & $1.8 \pm 07$ & $1.9 \pm 07$ & 0.477 \\
\hline CCS grading for angina & $1.6 \pm 1.1$ & $1.7 \pm 1.1$ & 0.496 \\
\hline Euroscore*(Q1-Q3) & $1.56(1.01-2.57)$ & $2.45(1.61-4.27)$ & 0.000 \\
\hline Diabetes & 28 & 30 & 0.108 \\
\hline LV Function & & & \\
Normal & 99 & 74 & \\
Moderate & 23 & 15 & \\
Poor & 0 & 2 & 0.974 \\
\hline Urgent & 9 & 14 & \\
Elective & 113 & 77 & 0.075 \\
\hline & & & \\
\hline
\end{tabular}

Table 2. Perioperative Variables of the C-ERAS and the Control Groups (Unadjusted)

\begin{tabular}{|c|c|c|c|}
\hline Details & C-ERAS(n=122) & Control $(n=91)$ & p-Value \\
\hline Bypass time (mins) & $84.9 \pm 26.2$ & $79.8 \pm 20.3$ & 0.133 \\
\hline Cross-clamp time(mins) & $59.3 \pm 17.6$ & $46.8 \pm 15.7$ & 0.000 \\
\hline Theatre time (mins) & & $219.9 \pm 73.8$ & .178 \\
\hline Temp on arrival at ICU $\left({ }^{\circ} \mathrm{C}\right)$ & $36.5 \pm 0.57$ & $35.9 \pm 0.55$ & 0.000 \\
\hline Ventilation Time (hours)* & $4.17(3.0,5.8)$ & $5.00(4.0,7.0)$ & 0.004 \\
\hline No of Grafts & $3.3 \pm 0.84$ & $2.8 \pm 0.71$ & 0.000 \\
\hline Cross-Clamp Time/Graft & $17.4 \pm 3.1$ & $16.6 \pm 4.5$ & 0.149 \\
\hline
\end{tabular}

Table 3. Post-operative complications and length of stay of the C-ERAS group and the Control Group (unadjusted)

\begin{tabular}{|l|l|l|l|}
\hline Details & C-ERAS $(\mathbf{n = 1 2 2})$ & Control $(\mathbf{n}=91)$ & p-Value \\
\hline ICU Stay (days) & $1.1 \pm 0.5$ & $1.4 \pm 0.5$ & 0.011 \\
\hline HDU Stay (days)* & $1.00(1.00,2.00)$ & $1.25(1.00,2.08)$ & 0.001 \\
\hline Hospital LOS (days)* & $7(6,8)$ & $8(7,11)$ & 0.000 \\
\hline Respiratory complications & 9 & 4 & 0.835 \\
\hline Acute Kidney Injury & 1 & 1 & 0.834 \\
\hline Arrythmias & 11 & 21 & 0.004 \\
\hline Other & 9 & 11 & 0.244 \\
\hline Reopened for bleeding & 1 & 1 & 0.834 \\
\hline
\end{tabular}

Table 4. Multivariable analysis after adjustment

\begin{tabular}{|l|c|c|c|}
\hline \multicolumn{1}{|c|}{ Multivariable analysis } & Mean & $\begin{array}{c}\text { 95\% Confidence } \\
\text { Interval }\end{array}$ & p-value \\
\hline $\begin{array}{l}\text { Difference in Length of Stay } \\
\text { (Days) }\end{array}$ & 2.36 & $1.01-3.7$ & $<0.01$ \\
\hline Bed Day Cost(f) & 1153.70 & $553.70-1753.70$ & $<0.01$ \\
\hline
\end{tabular}

\section{Discussion}

This study strongly elucidates the benefits of an enhanced recovery program (C-ERAS) in cardiac surgery. Earlyintervention in the pre-operative stage was highlighted by ERAS programmes in other surgical specialties that have been extrapolated to the cardiac surgery cohort. This includes early mobilisation, avoidance of prolonged fasting periods, early termination of intravenous fluids, earlier mobilization and enteral nutrition.

There are several challenges to implementing an enhanced recovery intervention. As an aggregation of minimal gains from each facet of care is needed for the success of a 'fast-tracked' pathway, a cohesive multidisciplinary team is obligatory to facilitate preoperative assessment, peri-operative care, intra-operative adjustments, post-operative care and follow-up post discharge. The importance of having an ERAS practitioner cannot be understated. ERAS practitioners provide training and ensure consistent implementation of care as well as auditing results. The significant change in practice may involve an increase in use of resources. However, this may be offset in the long run with shortened ICU and HDU stays as well as overall hospital stay, notwithstanding a reduction in post-operative complications. Schuster et al estimated that a simple intervention like gum chewing after colectomy could save $\$ 118828000$ per year in the United States ${ }^{13}$.

A measure of the health economic outcomes of an enhanced recovery programme however, has not been exhaustively examined. As there is a greater emphasis on care within the community, the benefits of may be overestimated if the cost creates a greater burden of care within the community or if there is a deterioration in quality of care in the community as opposed to conventional inhospital. 
Bernard et al conducted questionnaires on this matter and noted that post-discharge ERAS patients lacked appropriate support ${ }^{14}$. However, on commencement of a phone-in follow up clinic, majority of the patients in their colorectal cohort did not need additional input while convalescing and recovered well. The phone clinic therefore was probably more useful to patients in terms of offering reassurance and advice. It however cannot be assumed that an earlier discharge for ERAS patients equates to freedom from complications, or minor surgery. One potential reason for the perceived lack of postoperative support is the plethora of channels for a patient to access. The contact numbers available include general practitioners, district nurses, hospital ward telephone numbers, the ERAS practitioner and the nearest accident and emergency department. Blazeby et al highlighted that need for clear process for seeking help post discharge ${ }^{15}$. They conducted qualitative assessments of 20 patients from their enhanced recovery programmes following elective colonic resection to explore patients' experiences and views about the process. The main finding was the appreciation of a planned short hospital stay. However, some patients commented on feeling vulnerable at home shortly after major surgery.

One of the limitations of the study include the lack of long term follow up of patients in both cohorts. As mentioned above, the cost-benefit analysis of implementation of enhanced recovery programmes may shift the burden of care from the hospitals to the community and therefore be counter intuitive should there be complications.

This is one of the few studies to look at the implementation in ERAS patients outside a strictly elective list of patients in cardiac surgery. After a successful pilot with the elective cohort at our unit, we felt patients on the urgent list may also benefit from ERAS pathways. A nonrandomised matched proof of concept study in colorectal surgery based in Bangkok compared the outcomes of standard of care treatment and ERAS in a cohort of patients undergoing emergency surgery for acutely obstructed colorectal adenocarcinoma ${ }^{16}$. They concluded that ERAS was safe in the emergency setting with earlier discharge, earlier passing of flatus and time to resumption of normal diet and earlier initiation of adjuvant chemotherapy.

Despite this, ERAS is still currently in its infancy in cardiac surgery. To date, varying practices as well as preferences (on pump vs off-pump, total arterial vs single mammary, minimally invasive vs sternotomy and many more) may preclude a generic pathway for patients undergoing coronary artery bypass surgery. However with iterative learning, common benefits in the perioperative period may be universally applied to all centres for a multicentre study to truly investigate the benefits of ERAS.

\section{Conclusion}

This study highlighted that C-ERAS is a safe and feasible pathway to reduce in-hospital stay with no difference in complications and readmission rates compared to routine management of patients. There was also a significant cost saving with the C-ERAS pathway mimicking the results in enhanced recovery programmes in the other surgical specialities. A larger randomised multicentre study would be needed to allow greater generalisability of results. The bedrock of any enhanced recovery programme includes a cooperative multidisciplinary team of pre-admission staff, ERAS coordinator, physiotherapists, surgeons, anaesthetists, intensivists, nutritionists and nurses. All team members should be familiar with the ERAS ethos of the aggregations of minimal gains to a common goalnts and alter it accordingly.

\section{Acknowledgement}

We wish to thank Mr Balakrishnan Mahesh and Mr David Quinn for their support with the C-ERAS programme.

\section{References}

1. Kehlet H. Multimodal approach to control postoperative pathophysiology and rehabilitation. British journal of anaesthesia. 1997; 78(5):606-17. Epub 1997/05/01. PubMed PMID: 9175983.

2. Anderson ADG, McNaught CE, MacFie J, et al. Randomized clinical trial of multimodal optimization and standard perioperative surgical care. British Journal of Surgery. 2003; 90(12): 1497-504. doi: doi:10.1002/ bjs.4371.

3. Ljungqvist O, Scott M, Fearon KC. Enhanced Recovery After Surgery: A Review. JAMA surgery. 2017; 152(3): 292-8. Epub 2017/01/18. doi: 10.1001/jamasurg.2016.4952. PubMed PMID: 28097305.

4. Arumainayagam N, McGrath J, Jefferson KP, et al. Introduction of an enhanced recovery protocol for radical cystectomy. BJU international. 2008; 101(6): 698-701. Epub 2008/01/15. doi: 10.1111/j.1464410X.2007.07319.x. PubMed PMID: 18190646.

5. King PM, Blazeby JM, Ewings P, et al. The influence of an enhanced recovery programme on clinical outcomes, costs and quality of life after surgery for colorectal cancer. Colorectal disease : the official journal of the Association of Coloproctology of Great Britain and Ireland. 2006; 8(6): 506-13. Epub 2006/06/21. doi: 10.1111/j.14631318.2006.00963.x. PubMed PMID: 16784472.

6. Cerruto MA, De Marco V, D'Elia C, et al. Introduction of an enhanced recovery protocol to reduce short-term complications following radical cystectomy and intestinal urinary diversion with vescica ileale Padovana neobladder. Urologia internationalis. 2014; 92(1): 35-40. Epub 2013/09/21. doi: 10.1159/000351000. PubMed PMID: 24051504.

7. Zhuang CL, Ye XZ, Zhang XD, et al. Enhanced recovery after surgery programs versus traditional care for colorectal surgery: a metaanalysis of randomized controlled trials. Diseases of the colon and rectum. 2013; 56(5): 667-78. Epub 2013/04/12. doi: 10.1097/ DCR.0b013e3182812842. PubMed PMID: 23575408.

8. aradhan $\mathrm{KK}$, Neal KR, Dejong $\mathrm{CH}$, et al. The enhanced recovery after surgery (ERAS) pathway for patients undergoing major elective open colorectal surgery: a meta-analysis of randomized controlled trials. Clinical nutrition (Edinburgh, Scotland). 2010; 29(4): 434-40. Epub 2010/02/02. doi: 10.1016/j.clnu.2010.01.004. PubMed PMID: 20116145. 
9. Adamina $\mathrm{M}$, Kehlet $\mathrm{H}$, Tomlinson GA, et al. Enhanced recovery pathways optimize health outcomes and resource utilization: a meta-analysis of randomized controlled trials in colorectal surgery. Surgery. 2011; 149(6): 830-40. Epub 2011/01/18. doi: 10.1016/j. surg.2010.11.003. PubMed PMID: 21236454.

10. NiX,Jia D, Guo Y, et al. The efficacy and safety of enhanced recovery after surgery (ERAS) program in laparoscopic digestive system surgery: A meta-analysis of randomized controlled trials. International journal of surgery (London, England). 2019; 69: 108-15. Epub 2019/08/04. doi: 10.1016/j.ijsu.2019.07.034. PubMed PMID: 31376511.

11. Huddart S, Peden CJ, Swart M, et al. Use of a pathway quality improvement care bundle to reduce mortality after emergency laparotomy. The British journal of surgery. 2015; 102(1): 57-66. Epub 2014/11/12. doi: 10.1002/bjs.9658. PubMed PMID: 25384994; PubMed Central PMCID: PMCPmc4312892.

12. Mehta RL, Kellum JA, Shah SV, et al. Acute Kidney Injury Network: report of an initiative to improve outcomes in acute kidney injury. Critical care (London, England). 2007; 11(2): R31. Epub 2007/03/03. doi: 10.1186/cc5713. PubMed PMID: 17331245; PubMed Central PMCID: PMCPmc2206446.
13. Schuster R, Grewal N, Greaney GC, et al. Gum chewing reduces ileus after elective open sigmoid colectomy. Archives of surgery (Chicago, Ill : 1960). 2006; 141(2): 174-6. Epub 2006/02/24. doi: 10.1001/ archsurg.141.2.174. PubMed PMID: 16490895.

14. Bernard H, Foss M. The impact of the enhanced recovery after surgery (ERAS) programme on community nursing. British journal of community nursing. 2014; 19(4): 184, 6-8. Epub 2014/06/05. doi: 10.12968/bjcn.2014.19.4.184. PubMed PMID: 24893553.

15. Blazeby JM, Soulsby M, Winstone K, et al. A qualitative evaluation of patients' experiences of an enhanced recovery programme for colorectal cancer. Colorectal disease : the official journal of the Association of Coloproctology of Great Britain and Ireland. 2010; 12(10 Online): e236-42. Epub 2009/11/06. doi: 10.1111/j.14631318.2009.02104.x. PubMed PMID: 19888959.

16. Lohsiriwat V. Enhanced recovery after surgery vs conventional care in emergency colorectal surgery. World journal of gastroenterology. 2014; 20(38): 13950-5. Epub 2014/10/17. doi: 10.3748/wjg.v20. i38.13950. PubMed PMID: 25320532; PubMed Central PMCID: PMCPmc4194578. 